\title{
Mise en garde contre l'utilisation du produit MTP KIT présenté comme un médicament de l'interruption de grossesse et vendu sur Internet
}

\author{
Warning against the use of the MTP KIT product being sold as medical termination of pregnancy \\ on the Internet
}

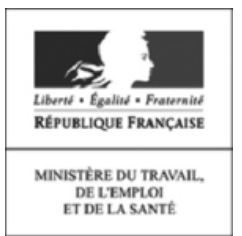

Direction générale de la santé - Afssaps

(C) Springer-Verlag France 2011

La Direction générale de la santé (DGS) et l'Agence française de sécurité sanitaire des produits de santé (Afssaps) souhaitent mettre en garde les consommateurs sur les risques graves pour la santé liés à l'utilisation d'un produit dénommé MTP KIT, présenté comme un médicament de l'IVG (interruption volontaire de grossesse) et vendu sur Internet.

Ce produit de la société CIPLA Limited est notamment vendu sur le site Eurodrugstore.eu sous la rubrique birth control ${ }^{1}$. Il s'agit d'un médicament illicite qui ne dispose d'aucune autorisation de mise sur le marché (AMM = autorisation octroyée par l'Afssaps après évaluation de la qualité, de la sécurité et de l'efficacité d'un médicament) ${ }^{1}$, ni d'autorisation d'importation sur le territoire français.

Les modalités d'emploi, notamment le mode d'administration du produit, exposent la femme qui l'utiliserait à un réel danger (échec de l'avortement, hémorragie, infection grave). En outre, les contrôles réalisés par les laboratoires de l'Afssaps ont montré que sa composition est inadaptée.

En France, une grande attention est apportée au droit d'accès à l'IVG pour toutes les femmes qui souhaitent y recourir et à la qualité de sa pratique, quel que soit le lieu

Direction générale de la santé

Mission information et communication,

14, avenue Duquesne, F-75007 Paris, France

\section{Afssaps}

143/147, boulevard Anatole-France,

F-93285 Saint-Denis cedex, France

\footnotetext{
${ }^{1}$ Contrôle des naissances.
}

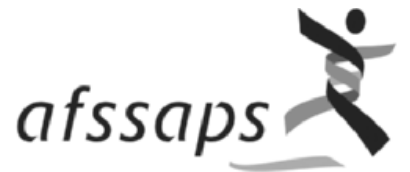

Agence française de sécurité sanitaire des produits de santé

de sa réalisation : établissement de santé, centre de planification familiale, centre de santé ou cabinet de ville. La pratique des IVG bénéficie d'un encadrement strict qui lui garantit de très bonnes conditions de sécurité et de prévention des complications.

Les informations sur les démarches à réaliser, les techniques d'IVG possibles, l'accompagnement psychosocial, les coordonnées des permanences téléphoniques régionales d'information sont apportées par le dossier-guide IVG : http://www.sante.gouv.fr/IMG/pdf/Guides_d'information sur_1_IVG_pour_les_femmes.pdf.

Ce document donne également des indications aux mineures souhaitant avoir accès à l'IVG dans des conditions d'anonymat et de gratuité.

Il faut enfin rappeler que certaines associations (par exemple, le Mouvement français pour le planning familial) aident les femmes placées dans une situation difficile, lorsqu'elles sont confrontées à une grossesse non désirée.

Par ailleurs, une information complète et didactique sur la contraception est disponible sur le site choisirsacontraception.fr.

La DGS et l'Afssaps rappellent qu'il n'est pas recommandé de se procurer des médicaments sur Internet ; leur provenance, leur qualité et leur sécurité n'étant pas garanties. L'achat de médicaments sur Internet comporte de nombreux risques pour la santé des consommateurs et peut favoriser leur mauvais usage. Seul le circuit des pharmacies d'officine est régulièrement contrôlé par les autorités sanitaires. 